\title{
Research Article \\ On the Frame Properties of Degenerate System of Sines
}

\author{
Bilal Bilalov and Fatima Guliyeva \\ Institute of Mathematics and Mechanics of NAS of Azerbaijan, B. Vahabzade 9, 1141 Baku, Azerbaijan \\ Correspondence should be addressed to Bilal Bilalov, bilalov.bilal@gmail.com
}

Received 27 June 2012; Accepted 17 August 2012

Academic Editor: Maria Isabel Berenguer

Copyright (c) 2012 B. Bilalov and F. Guliyeva. This is an open access article distributed under the Creative Commons Attribution License, which permits unrestricted use, distribution, and reproduction in any medium, provided the original work is properly cited.

Systems of sines with degenerate coefficients are considered in this paper. Frame properties of these systems in Lebesgue spaces are studied.

\section{Introduction}

Basis properties of classical system of exponents $\left\{e^{\mathrm{int}}\right\}_{n \in \mathbb{Z}}(\mathbb{Z}$ is the set of all integers) in Lebesgue spaces $L_{p}(-\pi, \pi), 1 \leq p<+\infty$, are well studied in the literature (see [1-4]). Bari in her fundamental work [5] raised the issue of the existence of normalized basis in $L_{2}$ which is not Riesz basis. The first example of this was given by Babenko [6]. He proved that the degenerate system of exponents $\left\{|t|^{\alpha} e^{\text {int }}\right\}_{n \in \mathbb{Z}}$ with $|\alpha|<(1 / 2)$ forms a basis for $L_{2}(-\pi, \pi)$ but is not Riesz basis when $\alpha \neq 0$. This result has been extended by Gaposhkin [7]. In [8], the condition on the weight $\rho$ was found which make the system $\left\{e^{\text {int }}\right\}_{n \in \mathbb{Z}}$ forms a basis for the weight space $L_{p, \rho}(-\pi, \pi)$ with a norm $\|f\|_{p, \rho}=\left(\int_{-\pi}^{\pi}|f(t)|^{p} \rho(t) d t\right)^{(1 / p)}$. Basis properties of a degenerate system of exponents are closely related to the similar properties of an ordinary system of exponents in corresponding weight space. In all the mentioned works, the authors consider the cases when the weight or the degenerate coefficient satisfies the Muckenhoupt condition (see, e.g., [9]). It should be noted that the above stated is true for the systems of sines and cosines, too.

Basis properties of the system of exponents and sines with the linear phase in weighted Lebesgue spaces have been studied in [10-12]. Those of the systems of exponents with degenerate coefficients have been studied in $[13,14]$. Similar questions have previously been considered in papers [15-18]. 
In this work, we study the frame properties of the system of sines with degenerate coefficient in Lebesgue spaces, when the degenerate coefficient, generally speaking, does not satisfy the Muckenhoupt condition.

\section{Needful Information}

To obtain our main results, we will use some concepts and facts from the theory of bases.

We will use the standard notation. $\mathbb{N}$ will be the set of all positive integers; $\exists$ will mean "there exist(s)"; $\Rightarrow$ will mean "it follows"; $\Leftrightarrow$ will mean "if and only if"; $\exists$ ! will mean "there exists unique"; $K \equiv \mathbb{R}$ or $K \equiv \mathbb{C}$ will stand for the set of real or complex numbers, respectively; $\delta_{n k}$ is Kronecker symbol, $\delta_{k}=\left\{\delta_{k n}\right\}_{k \in \mathbb{N}}$.

Let $X$ be some Banach space with a norm $\|\cdot\|_{X}$. Then $X^{*}$ will denote its dual with a norm $\|\cdot\|_{X^{*}}$. By $L[M]$, we denote the linear span of the set $M \subset X$, and $\bar{M}$ will stand for the closure of $M$.

System $\left\{x_{n}\right\}_{n \in \mathbb{N}} \subset X$ is said to be uniformly minimal in $X$ if $\exists \delta>0$

$$
\inf _{\forall u \in L\left[\left\{x_{n}\right\}_{n \neq k}\right]}\left\|x_{k}-u\right\|_{X} \geq \delta\left\|x_{k}\right\|_{X}, \quad \forall k \in \mathbb{N}
$$

System $\left\{x_{n}\right\}_{n \in \mathbb{N}} \subset X$ is said to be complete in $X$ if $\overline{L\left[\left\{x_{n}\right\}_{n \in \mathbb{N}}\right]}=X$. It is called minimal in $X$ if $x_{k} \notin \overline{L\left[\left\{x_{n}\right\}_{n \neq k}\right]}$, for all $k \in \mathbb{N}$.

The following criteria of completeness and minimality are available.

Criterion 1 (Hahn-Banach theorem). System $\left\{x_{n}\right\}_{n \in \mathbb{N}} \subset X$ is complete in $X$ if $f\left(x_{n}\right)=0$, for all $n \in \mathbb{N}, f \in X^{*} \Rightarrow f=0$.

Criterion 2 (see [19]). System $\left\{x_{n}\right\}_{n \in \mathbb{N}} \subset X$ is minimal in $X \Leftrightarrow$ it has a biorthogonal system $\left\{f_{n}\right\}_{n \in \mathbb{N}} \subset X^{*}$, that is, $f_{n}\left(x_{k}\right)=\delta_{n k}$, for all $n, k \in \mathbb{N}$.

Criterion 3. Complete system $\left\{x_{n}\right\}_{n \in \mathbb{N}} \subset X$ is uniformly minimal in $X \Leftrightarrow \sup _{n}\left\|x_{n}\right\|_{X}\left\|y_{n}\right\|_{X^{*}}<$ $+\infty$, where $\left\{y_{n}\right\}_{n \in \mathbb{N}} \subset X^{*}$ is a system biorthogonal to it. $\sum_{n=1}^{\infty} \lambda_{n} x_{n}$.

System $\left\{x_{n}\right\}_{n \in \mathbb{N}} \subset X$ is said to be a basis for $X$ if for all $x \in X, \exists !\left\{\lambda_{n}\right\}_{n \in \mathbb{N}} \subset K: x=$

If system $\left\{x_{n}\right\}_{n \in \mathbb{N}} \subset X$ forms a basis for $X$, then it is uniformly minimal.

Definition 2.1 (see $[20,21]$ ). Let $X$ be a Banach space and $\mathcal{K}$ a Banach sequence space indexed by $\mathbb{N}$. Let $\left\{f_{k}\right\}_{k \in \mathbb{N}} \subset X,\left\{g_{k}\right\}_{k \in \mathbb{N}} \subset X^{*}$. Then $\left(\left\{g_{k}\right\}_{k \in \mathbb{N}},\left\{f_{k}\right\}_{k \in \mathbb{N}}\right)$ is an atomic decomposition of $X$ with respect to $\mathcal{K}$ if

(i) $\left\{g_{k}(f)\right\}_{k \in \mathbb{N}} \in \mathcal{K}$, for all $f \in X$;

(ii) $\exists A, B>0$ :

$$
A\|f\|_{X} \leq\left\|\left\{g_{k}(f)\right\}_{k \in \mathbb{N}}\right\|_{\mathcal{K}} \leq B\|f\|_{X^{\prime}} \quad \forall f \in X ;
$$

(iii) $f=\sum_{k=1}^{\infty} g_{k}(f) f_{k}$, for all $f \in X$. 
Definition 2.2 (see $[20,21]$ ). Let $X$ be a Banach space and $\mathcal{K}$ a Banach sequence space indexed by $\mathbb{N}$. Let $\left\{g_{k}\right\}_{k \in \mathbb{N}} \subset X^{*}$ and $S: \mathcal{K} \rightarrow X$ be a bounded operator. Then $\left(\left\{g_{k}\right\}_{k \in \mathbb{N}}, S\right)$ is a Banach frame for $X$ with respect to $\mathcal{K}$ if

(i) $\left\{g_{k}(f)\right\}_{k \in \mathbb{N}} \in \mathcal{K}$, for all $f \in X$;

(ii) $\exists A, B>0$ :

$$
A\|f\|_{X} \leq\left\|\left\{g_{k}(f)\right\}_{k \in \mathbb{N}}\right\|_{\mathcal{K}} \leq B\|f\|_{X^{\prime}} \quad \forall f \in X ;
$$

(iii) $S\left[\left\{g_{k}(f)\right\}_{k \in \mathbb{N}}\right]=f$, for all $f \in X$.

It is true the following.

Proposition 2.3 (see $[20,21]$ ). Let $X$ be a Banach space and $\mathcal{K}$ a Banach sequence space indexed by $\mathbb{N}$. Assume that the canonical unit vectors $\left\{\delta_{k}\right\}_{k \in \mathbb{N}}$ constitute a basis for $\mathcal{K}$ and let $\left\{g_{k}\right\}_{k \in \mathbb{N}} \subset X^{*}$ and $S: \mathcal{K} \rightarrow X$ be a bounded operator. Then the following statements are equivalent:

(i) $\left(\left\{g_{k}\right\}_{k \in \mathbb{N}}, S\right)$ is a Banach frame for $X$ with respect to $\mathcal{K}$.

(ii) $\left(\left\{g_{k}\right\}_{k \in \mathbb{N}},\left\{S\left(\delta_{k}\right)\right\}_{k \in \mathbb{N}}\right)$ is an atomic decomposition of $X$ with respect to $\mathcal{K}$.

More details about these facts can be found in [20-23].

\section{Completeness and Minimality}

We consider a system of sines

$$
\left\{S_{n}^{v}\right\}_{n \in \mathbb{N}} \equiv\{v(t) \sin n t\}_{n \in \mathbb{N}}
$$

with a degenerate coefficient $v$

$$
v(t)=\prod_{k=0}^{r}\left|t-t_{k}\right|^{\alpha_{k}}
$$

where $0=t_{0}<t_{1}<\cdots<t_{r}=\pi$ are points of degeneration and $\left\{\alpha_{k}\right\}_{0}^{r} \subset R$.

The notation $f \sim g, t \rightarrow a$, means that the inequality $0<\delta \leq|f(t) / g(t)| \leq \delta^{-1}<+\infty$ holds in sufficiently small neighborhood of the point $t=a$ with respect to the functions $f$ and $g$. Thus, it is clear that $\sin n t \sim t, t \rightarrow 0$ and $\sin n t \sim \pi-t, t \rightarrow \pi$ for all $n \in \mathbb{N}$. Proceeding from these relations, we immediately obtain that the inclusion $\left\{S_{n}^{v}\right\}_{n \in \mathbb{N}} \subset L_{p}(0, \pi)$, $1 \leq p<+\infty$, is true if and only if the following relations hold

$$
\alpha_{0} ; \alpha_{r} \in\left(-\frac{1}{p}-1,+\infty\right), \quad\left\{\alpha_{k}\right\}_{1}^{r-1} \subset\left(-\frac{1}{p},+\infty\right)
$$


In what follows, we will always suppose that this condition is satisfied. Assume that the function $f \in L_{q}(0, \pi)((1 / p)+(1 / q)=1)$ is otrhogonal to the system $\left\{S_{n}^{v}\right\}_{n \in \mathbb{N}}$, that is

$$
\int_{0}^{\pi} v(t) \sin n t \bar{f}(t) d t=0, \quad \forall n \in \mathbb{N},
$$

where $(\cdot)$ is a complex conjugate. By $C_{0}[0, \pi]$, we denote the Banach space of functions which are continuous on $[0, \pi]$ with a sup-norm and vanish at the ends of the interval $[0, \pi]$. It is absolutely clear that $v f \in L_{1}(0, \pi) \subset C_{0}^{*}[0, \pi]$. As the system of sines $\{\sin n t\}_{n \in \mathbb{N}}$ is complete in $C_{0}[0, \pi]$, we obtain from the relations (3.4) that $v(t) f(t)=0$ a.e. on $(0, \pi)$, and, consequently, $f(t)=0$ a.e. on $(0, \pi)$. This proves the completeness of system (3.3) in $L_{p}(0, \pi)$.

Now consider the minimality of system (3.3) in $L_{p}(0, \pi)$. It is clear that $\left\{S_{n}^{v^{-1}}\right\}_{n \in \mathbb{N}} \subset$ $L_{q}(0, \pi)$ if and only if

$$
\alpha_{0} ; \alpha_{r} \in\left(-\infty, \frac{1}{q}+1\right), \quad\left\{\alpha_{k}\right\}_{1}^{r-1} \subset\left(-\infty, \frac{1}{q}\right)
$$

It is easily seen that the system $\left\{\sqrt{(2 / \pi)} S_{n}^{v^{-1}}\right\}_{n \in \mathbb{N}}$ is biorthogonal to $\left\{S_{n}^{v}\right\}_{n \in \mathbb{N}}$. So the following theorem is true.

Theorem 3.1. System $\left\{S_{n}^{v}\right\}_{n \in \mathbb{N}}$ is complete in $L_{p}(0, \pi), 1 \leq p<+\infty$, if the relations (3.3) hold. Besides, it is minimal in $L_{p}(0, \pi)$ if both (3.3) and (3.5) hold. Consequently, system $\left\{S_{n}^{v}\right\}_{n \in \mathbb{N}}$ is complete and minimal in $L_{p}(0, \pi)$ if the following relations hold:

$$
\alpha_{0} ; \alpha_{r} \in\left(-\frac{1}{p}-1, \frac{1}{q}+1\right), \quad\left\{\alpha_{k}\right\}_{1}^{r-1} \subset\left(-\frac{1}{p}, \frac{1}{q}\right) .
$$

It is known that (see, e.g., $[10,11])$ if $\left\{\alpha_{k}\right\}_{0}^{r} \subset(-(1 / p),(1 / q))$, then system $\left\{S_{n}(v)\right\}_{n \in \mathbb{N}}$ forms a basis for $L_{p}(0, \pi), 1<p<+\infty$. Let $\beta \in[(1 / q),(1 / q)+1)$, where either $\beta=\alpha_{0}$ or $\beta=\alpha_{r}$. In the sequel, we will suppose that the condition (3.6) is satisfied for $\left\{\alpha_{k}\right\}_{1}^{r-1}$. We have

$$
\left\|S_{n}^{v}\right\|_{p}^{p}=\int_{0}^{\pi}|v(t)|^{p}|\sin n t|^{p} d t \leq \int_{0}^{\pi}|v(t)|^{p} d t<+\infty, \quad \forall n \in \mathbb{N} .
$$

On the other hand

$$
\left\|S_{n}^{v}\right\|_{p}^{p} \geq \int_{0}^{\delta}|v(t)|^{p}|\sin n t|^{p} d t \geq c \int_{0}^{\delta} t^{\alpha_{0} p}|\sin n t|^{p} d t=\frac{c}{n^{1+\alpha_{0} p}} \int_{0}^{n \delta} t^{\alpha_{0} p}|\sin t|^{p} d t
$$

where $c>0$ is some constant (in what follows $c$ will denote constants that may be different from each other), $\delta>0$ is such that $[0, \delta]$ does not contain the points $\left\{\alpha_{k}\right\}_{1}^{r-1}$. Let us show that $\inf _{n \in \mathbb{N}}\left\|S_{n}^{v}\right\|_{p}>0$. We have $\left(\alpha=\alpha_{0} p+1\right)$

$$
\left\|S_{n}^{\nu}\right\|_{p}^{p} \geq \frac{c}{n^{\alpha}} \sum_{k \in M_{n}} \int_{k \pi+(\pi / 4)}^{(k+1) \pi-(\pi / 4)} t^{\alpha-1}|\sin t|^{p} d t \geq \frac{c}{n^{\alpha}} \sum_{k \in M_{n}} \int_{k \pi+(\pi / 4)}^{(k+1) \pi-(\pi / 4)} t^{\alpha-1} d t,
$$


where $M_{n} \equiv\{k \geq 0:(k+1) \pi-(\pi / 4) \leq n \delta\}$. Thus

$$
\left\|S_{n}^{v}\right\|_{p}^{p} \geq \frac{c}{n^{\alpha}} \sum_{k \in M_{n}} \int_{4 k+1}^{4 k+3} t^{\alpha-1} d t
$$

It is absolutely clear that

$$
\int_{4 k-1}^{4 k+1} t^{\alpha-1} d t \leq \int_{4 k+1}^{4 k+3} t^{\alpha-1} d t, \quad \forall k \in M_{n}
$$

Taking into account this relation, we obtain

$$
\left\|S_{n}^{v}\right\|_{p}^{p} \geq \frac{c}{n^{\alpha}} \sum_{k \in M_{n}}\left[\int_{4 k+1}^{4 k+3} t^{\alpha-1} d t+\int_{4 k-1}^{4 k+1} t^{\alpha-1} d t\right] \geq \frac{c}{n^{\alpha}} \int_{0}^{\lambda_{n}} t^{\alpha-1} d t=c\left(\frac{\lambda_{n}}{n}\right)^{\alpha}
$$

where $\lambda_{n} \geq(n \delta / \pi)-2$. Consequently

$$
\left\|S_{n}^{v}\right\|_{p}^{p} \geq c\left(\frac{\delta}{\pi}-\frac{2}{n}\right)^{\alpha} \longrightarrow c\left(\frac{\delta}{\pi}\right)^{\alpha}>0, \quad n \longrightarrow \infty .
$$

It follows immediately that $\inf _{n}\left\|S_{n}^{v}\right\|_{p}>0$.

Regarding biorthogonal system we get

$$
\left\|S_{n}^{-v}\right\|_{q}^{q}=\int_{0}^{\pi} v^{-q}(t)|\sin n t|^{q} d t .
$$

Choose $\varepsilon>0$ as small as the interval $[0, \varepsilon]$ does not contain the points $\left\{\alpha_{k}\right\}_{1}^{r-1}$. Consequently

$$
\left\|S_{n}^{-v}\right\|_{q}^{q}=m \int_{0}^{\varepsilon} t^{-\alpha_{0} q}|\sin n t|^{q} d t
$$

where $m>0$ is some constant. We have

$$
\left\|S_{n}^{-v}\right\|_{q}^{q} \geq m n^{\alpha_{0} q-1} \int_{0}^{n \varepsilon} \frac{|\sin t|^{q}}{t^{\alpha_{0}} q} d t
$$

First we consider the case $\alpha_{0} \in((1 / q),(1 / q)+1)$. In this case, for sufficiently great $n$, we have

$$
\left\|S_{n}^{-\nu}\right\|_{q}^{q} \geq m n^{\alpha_{0} q-1} \int_{0}^{1} \frac{|\sin t|^{q}}{t^{\alpha_{0} q}} d t \longrightarrow \infty, \quad n \longrightarrow \infty .
$$

Let $\alpha_{0}=(1 / q)$. Consequently

$$
\left\|S_{n}^{-v}\right\|_{q}^{q} \geq m \int_{0}^{n \varepsilon} \frac{|\sin t|^{q}}{t} d t
$$


and, as a result

$$
\begin{aligned}
\sup _{n}\left\|S_{n}^{-v}\right\|_{q}^{q} & \geq m \int_{1}^{+\infty} \frac{|\sin t|^{q}}{t} d t \geq m \sum_{k=1}^{\infty} \int_{k \pi+(\pi / 4)}^{(k+1) \pi-(\pi / 4)} \frac{|\sin t|^{q}}{t} d t \\
& \geq m c_{1} \sum_{k=1}^{\infty} \frac{1}{(k+1) \pi-(\pi / 4)} \int_{k \pi+(\pi / 4)}^{(k+1) \pi-(\pi / 4)} 1 d t=c_{2} \sum_{k=1}^{\infty} \frac{1}{(k+1) \pi-(\pi / 4)}=+\infty,
\end{aligned}
$$

where $c_{i}$ are some constants. So we obtain that for $\beta \in[(1 / q),(1 / q)+1), \sup _{n}\left\|S_{n}^{-v}\right\|_{q}=+\infty$. Consequently, in this case we have

$$
\sup _{n}\left\|S_{n}^{v}\right\|_{p}\left\|S_{n}^{-v}\right\|_{q}=+\infty
$$

Then it is known that (see, e.g., [22]) the system $\left\{S_{n}(\mathcal{v})\right\}_{n \in \mathbb{N}}$ is not uniformly minimal and, besides, does not form a basis for $L_{p}$.

Consider the case $\beta \in(-(1 / p)-1,-(1 / p)]$. Without limiting the generality, we will suppose that $\beta=\alpha_{0}$. In this case, with regard to the biorthogonal system we have

$$
\left\|S_{n}^{-v}\right\|_{q}^{q}=\int_{0}^{\pi} v^{-q}(t)|\sin n t|^{q} d t \leq \int_{0}^{\pi} v^{-q}(t) d t<+\infty, \quad \forall n \in \mathbb{N} .
$$

Taking sufficiently small $\delta>0$, we obtain

$$
\left\|S_{n}^{-\nu}\right\|_{q}^{q} \geq \int_{0}^{\delta} v^{-q}(t)|\sin n t|^{q} d t \geq c \int_{0}^{\delta} t^{-\alpha_{0} q}|\sin n t|^{q} d t .
$$

As $\alpha_{0} q<0$, then, in the absolutely same way as in the previous case, we get

$$
\inf _{n}\left\|S_{n}^{-v}\right\|_{q}>0
$$

On the other hand,

$$
\left\|S_{n}^{v}\right\|_{p}^{p}=\int_{0}^{\pi} v^{p}(t)|\sin n t|^{p} d t \geq c \int_{0}^{\delta} \frac{|\sin n t|^{p}}{t^{-\alpha_{0} p}} d t,
$$

where $\alpha_{0} p \leq 1$. Similarly to the previous case again, we get

$$
\int_{0}^{\delta} t^{\alpha_{0} p}|\sin n t|^{p} d t \longrightarrow+\infty, \quad n \longrightarrow \infty
$$

As a result we obtain

$$
\sup _{n}\left\|S_{n}^{v}\right\|_{p}\left\|S_{n}^{-v}\right\|_{q}=+\infty, \quad \text { for } \beta \in\left(-\frac{1}{p}-1,-\frac{1}{p}\right]
$$

Thus, the following theorem is true. 
Theorem 3.2. Let $\left\{\alpha_{0} ; \alpha_{r}\right\} \subset(-(1 / p)-1,-(1 / p)+2) ;\left\{\alpha_{0} ; \alpha_{r}\right\} \cap M_{p}^{(0)} \neq \emptyset$, and $A_{r} \subset$ $(-(1 / p),(1 / q))$, where $M_{p}^{(0)} \equiv(-(1 / p)-1,-(1 / p)] \bigcup[-(1 / p)+1,-(1 / p)+2), A_{r} \equiv\left\{\alpha_{k}\right\}_{1}^{r-1}$. Then the system $\left\{S_{n}^{v}\right\}_{n \in \mathbb{N}}$ is complete and minimal in $L_{p}(0, \pi), 1 \leq p<+\infty$, but does not form a basis for it.

\section{Defective Case}

Here, we consider the defective system of sines $\left\{S_{n}^{v}\right\}_{n \in \mathbb{N}_{\left(k_{0}\right)}}$, where $\mathbb{N}_{\left(k_{0}\right)} \equiv \mathbb{N} \backslash\left\{k_{0}\right\}, k_{0} \in \mathbb{N}$ is some number. It follows directly from Theorem 3.2 that if the condition

$$
\left\{\alpha_{0} ; \alpha_{r}\right\} \bigcap M_{p}^{(0)} \neq \emptyset ; \quad A_{r} \subset\left(-\frac{1}{p}, \frac{1}{q}\right)
$$

holds, then the system $\left\{S_{n}^{v}\right\}_{n \in \mathbb{N}_{\left(k_{0}\right)}}$ is minimal but not complete in $L_{p}(0, \pi), 1 \leq p<+\infty$. Assume $M_{p}^{(1)} \equiv[(1 / q)+1,(1 / q)+3)$. Let $\alpha_{0} \in M_{p}^{(1)}$. Consider the completeness of system $\left\{S_{n}^{v}\right\}_{n \in \mathbb{N}_{\left(k_{0}\right)}}$ in $L_{p}(0, \pi)$. Suppose that $f \in L_{q}(0, \pi)$ is orthogonal to the system, that is,

$$
\int_{0}^{\pi} v(t) \sin n t \overline{f(t)} d t=0, \quad \forall n \in \mathbb{N}_{\left(k_{0}\right)}
$$

As $v f \in L_{1} \subset C_{0}^{*}[0, \pi]$ and system $\{\sin n t\}_{n \in \mathbb{N}}$ is complete and minimal in $C_{0}[0, \pi]$, from (4.2) we get

$$
v(t) f(t)=c \sin k_{0} t \Longrightarrow f(t)=c \frac{\sin k_{0} t}{v(t)}
$$

It is clear that $v^{-1}(t) \sim t^{-\alpha_{0}}, \sin k_{0} t \sim t$ as $t \rightarrow 0$. Consequently, $f \sim t^{-\alpha_{0}+1}, t \rightarrow 0$. As $q\left(-\alpha_{0}+1\right) \leq-1$, then $f \in L_{q}(0, \pi)$ if and only if $c=0$, and, consequently, $f=0$. The similar result is true for $\alpha_{r} \in M_{p}^{(1)}$. Thus, if $\alpha_{0} ; \alpha_{r}>-(1 / p)-1$, and $\max \left\{\alpha_{0} ; \alpha_{r}\right\} \in M_{p}^{(1)}$, then the system $\left\{S_{n}^{v}\right\}_{n \in \mathbb{N}_{\left(k_{0}\right)}}$ is complete in $L_{p}(0, \pi)$. Now we consider the minimality of this system. Let

$$
\vartheta_{n}(t)=\mathcal{v}^{-1}(t)\left[\frac{\sin n x}{n}-\frac{\sin k_{0} x}{k_{0}}\right], \quad n \in \mathbb{N}_{\left(k_{0}\right)}
$$

We have

$$
\left\langle S_{k}^{v}, \vartheta_{n}\right\rangle=\int_{0}^{\pi} S_{k}^{v}(t) \overline{\vartheta_{n}(t)} d t=\frac{\pi}{2 n} \delta_{k n}, \quad \forall k, n \in \mathbb{N}_{\left(k_{0}\right)}
$$


Let us show that $\left\{\vartheta_{n}\right\}_{n \in \mathbb{N}_{\left(k_{0}\right)}} \subset L_{q}(0, \pi)$. In fact

$$
\begin{aligned}
& \frac{\sin n t}{n}=t-\frac{n^{2} t^{3}}{6}+o\left(t^{3}\right), \quad t \longrightarrow 0, \\
& \frac{\sin k_{0} t}{k_{0}}=t-\frac{k_{0}^{2} t^{3}}{6}+o\left(t^{3}\right), \quad t \longrightarrow 0,
\end{aligned}
$$

and, consequently

$$
\frac{\sin n t}{n}-\frac{\sin k_{0} t}{k_{0}}=\frac{1}{6}\left(k_{0}^{2}-n^{2}\right) t^{3}+o\left(t^{3}\right), \quad t \rightarrow 0 .
$$

From these relations, we immediately find that $\vartheta_{n}(t) \sim t^{3-\alpha_{0}}, t \rightarrow 0$. As a result, $\left\{\vartheta_{n}\right\}_{n \in \mathbb{N}_{\left(k_{0}\right)}} \subset$ $L_{q}(0, \pi)$. Then the relations (4.5) imply the minimality of system $\left\{S_{n}^{v}\right\}_{n \in \mathbb{N}_{\left(k_{0}\right)}}$ in $L_{p}(0, \pi)$. Similar result is true for $\alpha_{r} \in M_{p}^{(1)}$. In the end, we obtain that if $\max \left\{\alpha_{0} ; \alpha_{r}\right\} \in M_{p}^{(1)}$, then the system $\left\{S_{n}^{v}\right\}_{n \in \mathbb{N}}$ has a defect equal to 1 .

Consider the case when $\max \left\{\alpha_{0} ; \alpha_{r}\right\}=\alpha_{0} \in M_{p}^{(2)}$, where $M_{p}^{(2)} \equiv[(1 / q)+3,(1 / q)+5)$. We look at the system $\left\{S_{n}^{v}\right\}_{n \in \mathbb{N}_{\left(k_{1} ; k_{2}\right)}}$, where $\mathbb{N}_{\left(k_{1} ; k_{2}\right)} \equiv \mathbb{N} \backslash\left\{k_{1} ; k_{2}\right\}, \quad k_{1} ; k_{2} \in \mathbb{N}, k_{1} \neq k_{2}$, are some numbers. Let $f \in L_{q}(0, \pi)$ cancel this system out, that is,

$$
\left\langle S_{n}^{v}, f\right\rangle=0, \quad \forall n \in \mathbb{N}_{\left(k_{1} ; k_{2}\right)} .
$$

Using the previous reasoning, we find that for some constants $c_{1}, c_{2}$, the following is true:

$$
f(t)=c_{1} v^{-1}(t) \sin k_{1} t+c_{2} v^{-1}(t) \sin k_{2} t .
$$

Using representations

$$
\sin k_{i} t=k_{i} t-\frac{k_{i}^{3}}{6} t^{3}+o\left(t^{4}\right), \quad t \longrightarrow 0, i=1,2,
$$

we obtain

$$
f(t) \sim c t^{-\alpha_{0}} g_{1}(t)+g_{2}(t), \quad t \longrightarrow 0
$$

( $c \neq 0$ is some constant), where it can be easily seen that $g_{2} \in L_{q}$ and

$$
g_{1}(t)=\left(c_{1} k_{1}+c_{2} k_{2}\right) t-\frac{1}{6}\left(c_{1} k_{1}^{3}+c_{2} k_{2}^{3}\right) t^{3}
$$

Thus, $f \in L_{q}$ if and only if $t^{-\alpha_{0}} g_{1}(t) \in L_{q}$. Assume $b_{1}=c_{1} k_{1}+c_{2} k_{2} ; b_{2}=-(1 / 6)\left(c_{1} k_{1}^{3}+c_{2} k_{2}^{3}\right)$. As $\left(1-\alpha_{0}\right) q \leq-1\left(3-\alpha_{0}\right) q \leq-1$, it is clear that $t^{1-\alpha_{0}} \notin L_{q}$ and $t^{3-\alpha_{0}} \notin L_{q}$. Suppose $b_{1} \neq 0$. We have

$$
\left|t^{-\alpha_{0}} g_{1}(t)\right|=\left|b_{1}\right| t^{1-\alpha_{0}}\left|1+b_{1}^{-1} b_{2} t^{2}\right|
$$


It follows directly that for sufficiently small $\delta>0$, we have

$$
\left|t^{-\alpha_{0}} g_{1}(t)\right| \geq c_{\delta}\left|b_{1}\right| t^{1-\alpha_{0}}, \quad \forall t \in(0, \delta)
$$

where $c_{\delta}>0$ is some constant depending only on $\delta$ and $b_{2}$. As a result, $f \notin L_{q}$.

Consequently, $b_{1}=0$. Moreover, it is not difficult to derive that $b_{2}=0$. Thus, we obtain the following system for $c_{1}$ and $c_{2}$ :

$$
\begin{aligned}
& c_{1} k_{1}+c_{2} k_{2}=0, \\
& c_{1} k_{1}^{3}+c_{2} k_{2}^{3}=0 .
\end{aligned}
$$

It is clear that $\operatorname{det}\left|\begin{array}{ll}k_{1} & k_{2} \\ k_{1}^{3} & k_{2}^{3}\end{array}\right| \neq 0$. And, consequently, $c_{1}=c_{2}=0$. As a result, $f=0$, which, in turn, implies that the system $\left\{S_{n}^{v}\right\}_{n \in \mathbb{N}_{\left(k_{1} ; k_{2}\right)}}$ is complete in $L_{p}$. Let us show that it is also minimal in $L_{p}$. Assume $\gamma_{n}^{(k)}=(1 / 6)\left(k^{2}-n^{2}\right)$. Consider the system $\vartheta_{n}^{\left(k_{1} ; k_{2}\right)}(t) \equiv\left[a_{n}^{\left(k_{1} ; k_{2}\right)} \sin n t-\right.$ $\left.\left(1 / k_{1} \gamma_{n}^{\left(k_{1}\right)}\right) \sin k_{1} t+\left(1 / k_{2} \gamma_{n}^{\left(k_{2}\right)}\right) \sin k_{2} t\right] v^{-1}(t)$, for all $n \in \mathbb{N}_{\left(k_{1} ; k_{2}\right)}$, where

$$
a_{n}^{\left(k_{1} ; k_{2}\right)}=\frac{1}{n}\left(\frac{1}{\gamma_{n}^{\left(k_{1}\right)}}-\frac{1}{\gamma_{n}^{\left(k_{2}\right)}}\right) \neq 0
$$

Simple calculations give the following representation:

$$
\vartheta_{n}^{\left(k_{1} ; k_{2}\right)}(t) \sim c_{n} t^{5}, \quad t \longrightarrow 0,
$$

where $c_{n} \neq 0$, for all $n \in \mathbb{N}_{\left(k_{1} ; k_{2}\right)}$ are some constants. We obtain directly from this representation that $\left\{\vartheta_{n}^{\left(k_{1} ; k_{2}\right)}\right\}_{n \in \mathbb{N}\left(k_{1} ; k_{2}\right)} \subset L_{q}$. On the other hand,

$$
\left\langle S_{n}^{v}, \vartheta_{n}^{\left(k_{1} ; k_{2}\right)}\right\rangle=a_{n}^{\left(k_{1} ; k_{2}\right)} \delta_{n k}=\left\{\begin{array}{l}
\neq 0, n \neq k, \\
=0, n=k,
\end{array} \quad \forall n, k \in \mathbb{N}_{\left(k_{1} ; k_{2}\right)}\right.
$$

Thus, if $\alpha_{0} \in M_{p}^{(2)}$, then the system $\left\{S_{n}^{v}\right\}_{n \in \mathbb{N}_{\left(k_{1} ; k_{2}\right)}}$ is complete and minimal in $L_{p}$, and, as a result, the system $\left\{S_{n}^{v}\right\}_{n \in \mathbb{N}}$ has a defect equal to 2 . It is easy to see that the similar result is true if $\alpha_{r} \in M_{p}^{(2)}$ with $\alpha_{0} \leq \alpha_{r}$. Continuing this way, we obtain that if $\beta=\max \left\{\alpha_{0} ; \alpha_{r}\right\}=\alpha_{0} \in M_{p}^{(k)}$, where $M_{p}^{(k)} \equiv[-(1 / p)+2 k,-(1 / p)+2(k+1))$, then the system $\left\{S_{n}^{v}\right\}_{n \in \mathbb{N}_{\left\{\bar{n}_{k}\right\}}}$ is complete and minimal in $L_{p}$, where $\left\{\bar{n}_{k}\right\}=\left\{n_{1} ; \ldots ; n_{k}\right\} \subset \mathbb{N}, n_{i} \neq n_{j}$ with $i \neq j$.

Consider the basicity of system $\left\{S_{n}^{v}\right\}_{n \in \mathbb{N}_{\left(\bar{n}_{1}\right\}}}$ (i.e., the case of $k=1$ ) in $L_{p}$. Similar to the case of $M_{p}^{(0)}$, it can be proved that

$$
0<\inf _{n \in \mathbb{N}\left\{\bar{n}_{1}\right\}}\left\|S_{n}^{v}\right\|_{p} \leq \sup _{n \in \mathbb{N}_{\left\{\bar{n}_{1}\right\}}}\left\|S_{n}^{v}\right\|_{p}<+\infty
$$


Concerning biorthogonal system, we have

$$
\int_{0}^{\pi} v^{-q}(x)\left|\frac{\sin n x}{n}-\frac{\sin k_{1} x}{k_{1}}\right|^{q} d x \geq c \int_{0}^{\varepsilon} x^{-q \alpha_{0}}\left|\frac{\sin n x}{n}-\frac{\sin n_{1} x}{n_{1}}\right|^{q} d x,
$$

where the interval $[0, \varepsilon](\varepsilon>0)$ does not contain the points $\left\{t_{k}\right\}_{1}^{r-1}$. As $\left(\sin n_{1} x / n_{1}\right)-x \sim x^{3}$, for $x \rightarrow 0$, it is clear that $\left(-q \alpha_{0}+3>-1\right)$

$$
\int_{0}^{\varepsilon} x^{-q \alpha_{0}}\left|\frac{\sin n_{1} x}{n_{1}}-x\right|^{q} d x<+\infty
$$

Taking this circumstance into account, we have

$$
\left\|S_{n}^{-v}\right\|_{q}^{q} \geq c\left[\int_{0}^{\varepsilon} x^{-q \alpha_{0}}\left|\frac{\sin n x}{n}-x\right|^{q} d x-\int_{0}^{\varepsilon} x^{-q \alpha_{0}}\left|\frac{\sin n_{1} x}{n_{1}}-x\right|^{q} d x\right] .
$$

Consider the case of $\alpha_{0} \in((1 / q)+1,(1 / q)+3)$ :

$$
\begin{aligned}
\int_{0}^{\varepsilon} x^{-q \alpha_{0}}\left|\frac{\sin n x}{n}-x\right|^{q} d x & =n^{-q} \int_{0}^{\varepsilon} x^{-q \alpha_{0}}|\sin n x-n x|^{q} d x \\
& =n^{-\alpha} \int_{0}^{n \varepsilon} t^{-q \alpha_{0}}|\sin n t-t|^{q} d t \\
& \geq c n^{-\alpha} \int_{0}^{n \varepsilon} t^{-q \alpha_{0}+q} d t=\frac{c}{\alpha}\left(\varepsilon^{\alpha}-n^{-\alpha}\right),
\end{aligned}
$$

where $\alpha=-\alpha_{0} q+q+1<0$. Consequently, $\sup _{n}\left\|S_{n}^{-\nu}\right\|_{q}=+\infty$. Let $\alpha_{0}=(1 / q)+1$. In this case we have

$$
\int_{0}^{\varepsilon} x^{-q \alpha_{0}}\left|\frac{\sin n x}{n}-x\right|^{q} d x \geq c \int_{1}^{n \varepsilon} t^{-1} d t=c \ln n \varepsilon
$$

and, consequently $\sup _{n}\left\|S_{n}^{-v}\right\|_{q}=+\infty$. As a result, we get that for $\alpha_{0} \in M_{p}^{(1)}$, the system $\left\{S_{n}^{v}\right\}_{n \in \mathbb{N}_{\left\{\bar{n}_{1}\right\}}}$ does not form a basis for $L_{p}$. Assume that in this case, the system $\left\{S_{n}^{v}\right\}_{n \in \mathbb{N}}$ is a frame in $L_{p}$, that is any function from $L_{p}$ can be expanded with respect to this system. As it does not form a basis for $L_{p}$, zero has a non trivial decomposition, that is,

$$
0=\sum_{n=1}^{\infty} a_{n} S_{n}^{v}
$$

where $\exists n_{0} \in \mathbb{N}: a_{n_{0}} \neq 0$. As the system $\left\{S_{n}^{v}\right\}_{n \in \mathbb{N}_{\left(\bar{n}_{1} \mid\right.}}$ is complete and minimal in $L_{p}$, it is clear that $a_{n_{1}} \neq 0$. Consequently, $S_{n_{1}}^{v}=\sum_{n \neq n_{1}}\left(a_{n} / a_{n_{1}}\right) S_{n}^{v}$. It follows directly that the arbitrary element can be expanded with respect to the system $\left\{S_{n}^{v}\right\}_{n \in \mathbb{N}_{\left(\bar{n}_{1}\right]}}$. But this is impossible. Similar result is true for $\max \left\{\alpha_{0} ; \alpha_{r}\right\} \in M_{p}^{(1)}$. 
Proceeding in an absolutely similar way as we did in the previous case, we can prove that for $\max \left\{\alpha_{0} ; \alpha_{r}\right\} \in M_{p}^{(k)}$, the system $\left\{S_{n}^{v}\right\}_{n \in \mathbb{N}_{\left\{\bar{n}_{k} \mid\right.}}$ is complete and minimal in $L_{p}$, but does not form a basis for it. Consequently, system $\left\{S_{n}^{\nu}\right\}_{n \in \mathbb{N}}$ has a defect equal to $(k)$. The fact that it is not a frame in $L_{p}$ in this case too is proved as follows. Let $k=2:\left\{\bar{n}_{k}\right\} \equiv\left\{n_{1} ; n_{2}\right\}$. Assume that the system $\left\{S_{n}\right\}_{n \in \mathbb{N}}$ is a frame in $L_{p}$. Then zero has a non trivial decomposition: $0=\sum_{n=1}^{\infty} a_{n} S_{n}^{v}$. It is clear that $\left|a_{n_{1}}\right|+\left|a_{n_{2}}\right|>0$, and let $a_{n_{1}} \neq 0$. It follows directly that the system $\left\{S_{n}\right\}_{n \neq n_{1}}$ is a frame in $L_{p}$. The further reasoning is absolutely similar to the case of $k=1$. This scheme is applicable for for all $k \in \mathbb{N}$. Thus, we have proved the following main theorem.

Theorem 4.1. Let the following necessary condition be satisfied

$$
\alpha_{0} ; \alpha_{r} \in\left(-\frac{1}{p}-1,+\infty\right), \quad\left\{\alpha_{k}\right\}_{1}^{r-1} \subset\left(-\frac{1}{p} ; \frac{1}{q}\right) .
$$

Then the system $\left\{S_{n}^{v}\right\}_{n \in \mathbb{N}}$ is a frame (basis) in $L_{p}$ if and only if $\alpha_{0} ; \alpha_{r} \in(-(1 / p),(1 / q))$. Moreover, for $\max \left\{\alpha_{0} ; \alpha_{r}\right\} \in M_{p}^{(k)}, k \in \mathbb{N}$, it has a defect equal to $(k)$, where $M_{p}^{(k)} \equiv[(1 / q)+$ $2 k,(1 / q)+2(k+1))$.

\section{Acknowledgment}

The authors are thankful to the referees for their valuable comments.

\section{References}

[1] R. Edwards, Fourier Series in A Modern Exposition, vol. 1, Mir, Moscow, Russia, 1985.

[2] R. Edwards, Fourier Series in A Modern Exposition, vol. 2, Mir, Moscow, Russia, 1985.

[3] A. Zigmund, Trigonometric Series, vol. 1, Mir, Moscow, Russia, 1965.

[4] A. Zigmund, Trigonometric Series, vol. 2, Mir, Moscow, Russia, 1965.

[5] N. K. Bari, Trigonometric Series, Fizmatgiz, Moscow, Russia, 1961.

[6] K. I. Babenko, "On conjugate functions," Doklady Akademii Nauk SSSR, vol. 62, no. 2, pp. 157-160, 1948.

[7] V. F. Gaposhkin, "One generalization of M.Riesz theorem on conjugated functions," Matematicheskii Sbornik, vol. 46(88), no. 3, pp. 359-372, 1958.

[8] K. S. Kazaryan and P. I. Lizorkin, "Multipliers, bases and unconditional bases of the weighted spaces $B$ and SB," Proceedings of the Steklov Institute of Mathematics, vol. 187, pp. 111-130, 1989.

[9] J. Garnett, Bounded Analytic Functions, Mir, Moscow, Russia, 1984.

[10] E. I. Moiseev, "On basicity of systems of cosines and sines in weight space," Differentsial'nye Uravneniya, vol. 34, no. 1, pp. 40-44, 1998.

[11] E. I. Moiseev, "The basicity in the weight space of a system of eigen functions of a differential operator," Differentsial'nye Uravneniya, vol. 35, no. 2, pp. 200-205, 1999.

[12] S. S. Pukhov and A. M. Sedletskiı̌, "Bases of exponentials, sines, and cosines in weighted spaces on a finite interval," Rossiǔskaya Akademiya Nauk. Doklady Akademii Nauk, vol. 425, no. 4, pp. 452-455, 2009.

[13] B. T. Bilalov and S. G. Veliyev, "On completeness of exponent system with complex coefficients in weight spaces," Transactions of NAS of Azerbaijan, vol. 25, no. 7, pp. 9-14, 2005.

[14] B. T. Bilalov and S. G. Veliev, "Bases of the eigenfunctions of two discontinuous differential operators," Differentsial'nye Uravneniya, vol. 42, no. 10, pp. 1503-1506, 2006.

[15] B. T. Bilalov, "Bases of exponentials, cosines, and sines that are eigenfunctions of differential operators," Differentsial'nye Uravneniya, vol. 39, no. 5, pp. 652-657, 2003.

[16] B. T. Bilalov, "On basicity of some systems of exponents, cosines and sines," Rossiǔskaya Akademiya Nauk. Doklady Akademii Nauk, vol. 379, no. 2, pp. 7-9, 2001. 
[17] B. T. Bilalov, "Basis properties of some systems of exponentials, cosines, and sines," Sibirskiu Matematicheskiu Zhurnal, vol. 45, no. 2, pp. 264-273, 2004.

[18] E. S. Golubeva, "The System of weighted exponentials with power weights. Herald of Samara State University," Natural Sciences, vol. 83, no. 2, pp. 15-25, 2011.

[19] J. Lindenstrauss and L. Tzafriri, Classical Banach Spaces, Springer, 1996.

[20] O. Christensen, An Introduction to Frames and Riesz Bases, Springer, 2003.

[21] A. Rahimi, Frames and Their Generalizations in Hilbert and Banach Spaces, Lambert Academic Publishing, 2011.

[22] C. Heil, A Basis Theory Primer, Springer, 2011.

[23] M. G. Krein, Functional Analysis, Nauka, Moscow, Russia, 1972. 


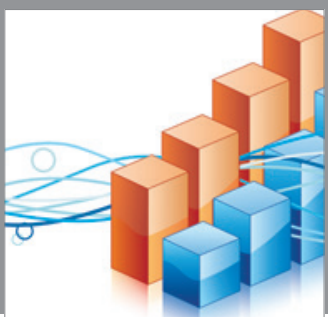

Advances in

Operations Research

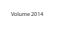

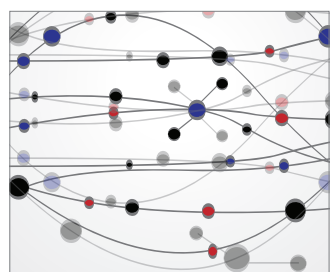

\section{The Scientific} World Journal
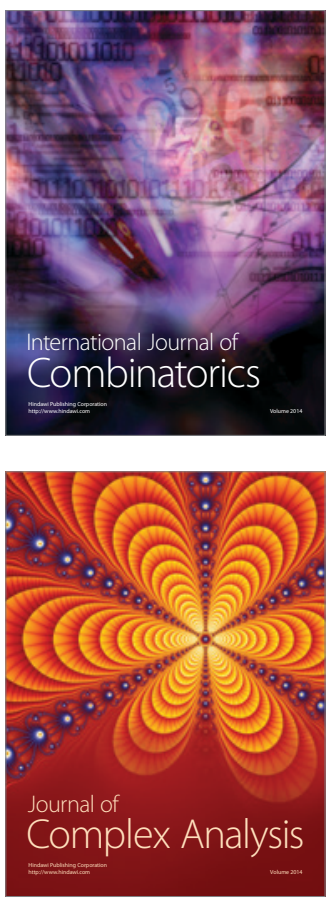

International Journal of

Mathematics and

Mathematical

Sciences
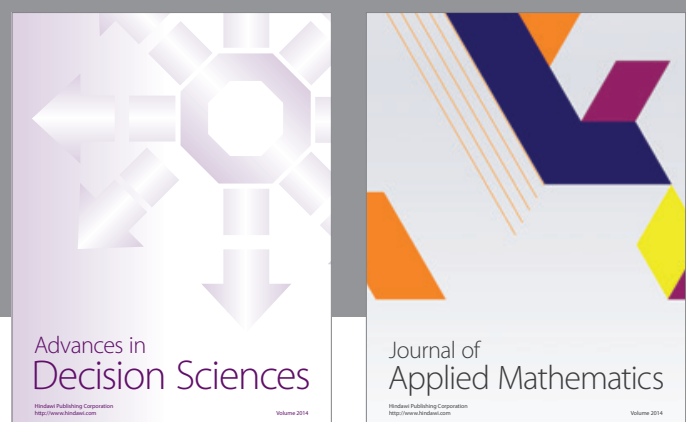

Journal of

Applied Mathematics
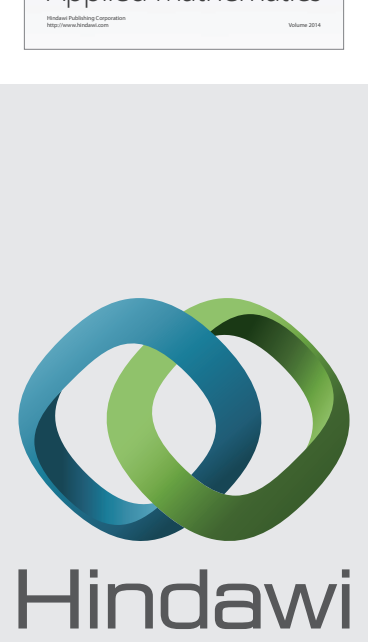

Submit your manuscripts at http://www.hindawi.com
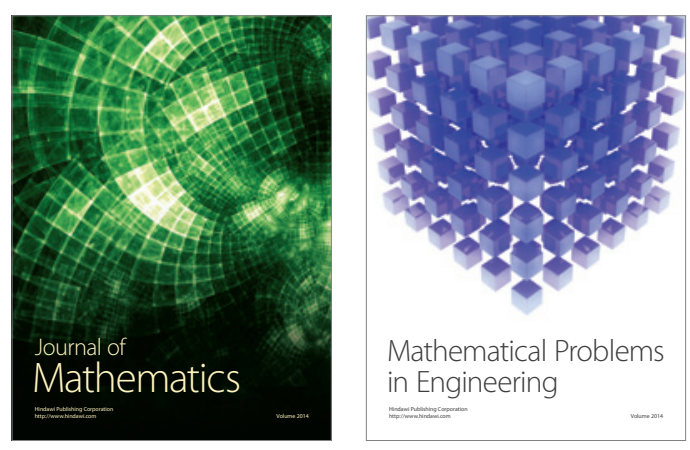

Mathematical Problems in Engineering
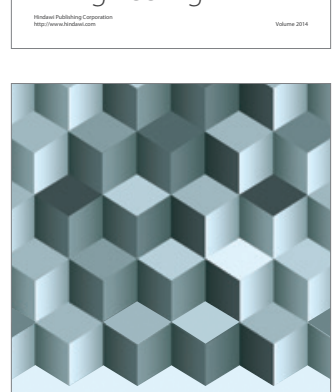

Journal of

Function Spaces
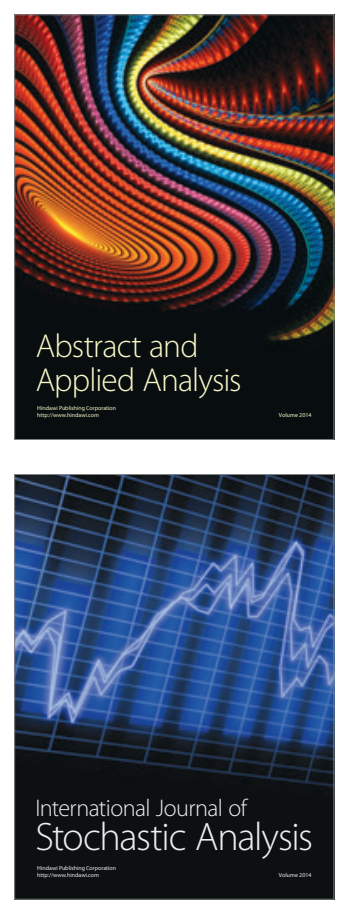

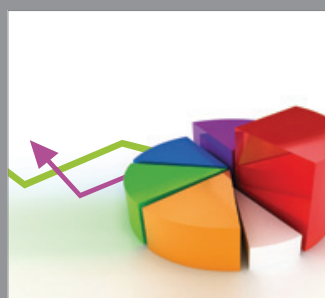

ournal of

Probability and Statistics

Promensencen
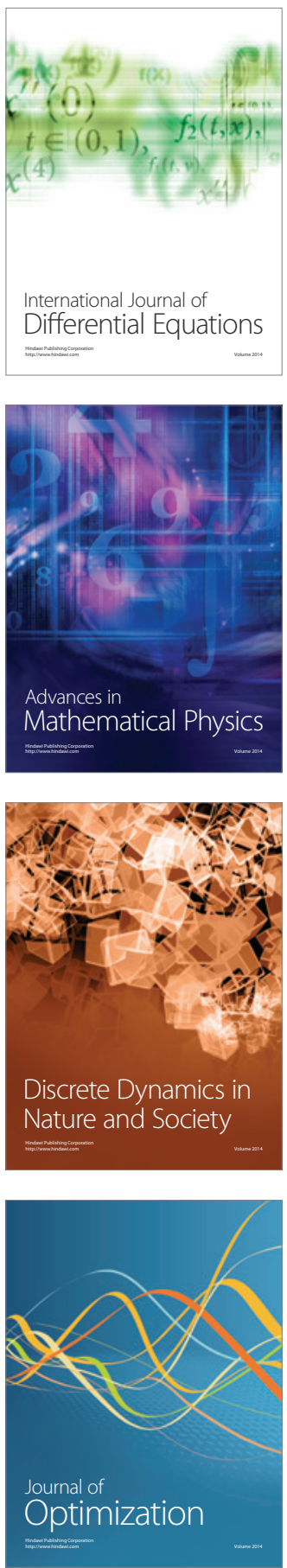